Rev. Int. Contam. Ambie. 35 (1) 151-163, 2019

DOI: 10.20937/RICA.2019.35.01.11

\title{
ANÁLISIS ECOTOXICOLÓGICO DEL FUNGICIDA FOSFITO DE SODIO SOBRE UNA COMUNIDAD PLANCTÓNICA
}

\author{
Gema PARRA ANGUITA*, Valeria ACEVEDO-GARCÍA, Ma. Eugenia LÓPEZ VALCARCEL, \\ y Ana Isabel DEL ARCO OCHOA
}

Departamento de Biología Animal, Biología Vegetal y Ecología, Universidad de Jaén, Campus Las Lagunillas s/n, 23071 Jaén, España

* Autor para correspondencia gparra@ujaen.es

(Recibido enero 2018; aceptado mayo 2018)

Palabras clave: ecotoxicología, microcosmos, zooplancton, agroquímico

\section{RESUMEN}

La agricultura es una actividad estratégica de la sociedad que ocasiona impactos negativos sobre el ambiente, algunos relacionados con el uso excesivo de productos de síntesis para el control de plagas o la fertilización química. El fungicida fosfito de sodio presenta datos de baja toxicidad bajo condiciones estándar de laboratorio y organismos tipo ensayo. Sin embargo, no se han realizado ensayos ecotoxicológicos que permitan ayudar en la evaluación del riesgo ambiental de esta sustancia. El presente trabajo estudia el efecto de la exposición de larga duración (60 días) del fungicida fosfito de sodio sobre la comunidad planctónica, a través de ensayos con microcosmos. Para la determinación de las concentraciones a utilizar en los microcosmos se realizó un ensayo preliminar uniespecífico crónico de exposición con Daphnia magna en laboratorio. Después de evaluar los resultados de dicho experimento, se decidió que las concentraciones de exposición en los microcosmos fueran 5 y $10 \mathrm{mg} / \mathrm{L}$. Los resultados de los microcosmos indican que no han tenido lugar efectos negativos, asociado al tratamiento en términos estructurales (abundancia y composición taxonómica) de la comunidad zooplanctónica. Además, la comunidad de productores primarios, fitoplancton y perifiton, tampoco mostró respuesta negativa, en términos de cambios en abundancia, a dicha exposición. Los resultados indican que la utilización de fosfito a estas concentraciones no representa un riesgo para la comunidad zooplanctónica bajo estudio. Sin embargo, la falta de información sobre los efectos en la estructura del componente fitoplanctónico debe hacer primar el principio de precaución en el uso de esta sustancia.

Key words: ecotoxicology, microcosms, zooplankton, agrochemical

\begin{abstract}
Agriculture is a strategic activity for the society, capable of causing impacts on the environment. Most of the impacts are related to the overuse of synthetic products for pest control and/or chemical fertilization. Sodium phosphite used as a fungicide presents low toxicity under standard laboratory conditions and type test organisms. However, no ecotoxicological tests have been performed to assist in the environmental risk
\end{abstract}


assessment of this substance within a holistic framework. The present study shows the effect of the long-term exposure (60 days) of the fungicide sodium phosphite on the planktonic community, through outdoor microcosm tests. To determine the convenient concentrations to be used in the microcosms, a preliminary monospecific test was carried out with Daphnia magna in the laboratory. Considering its results, the levels of exposure to the microcosms were 5 and $10 \mathrm{mg} / \mathrm{L}$. The results have shown no effect on the zooplankton community structure (taxonomic composition or abundance) associated with treatments. In addition, the community of primary producers, phytoplankton and periphyton, also showed negative response (abundance) under the exposure conditions. Present results indicate that the use of sodium phosphite at these concentrations does not represent a risk for the zooplankton community, however the lack of information about the effects on the phytoplankton structure should make the precautionary principle prevail in the use of this substance.

\section{INTRODUCCIÓN}

La agricultura es una actividad indispensable para el bienestar humano, puesto que proporciona productos básicos para el sustento alimentario y determina parte de la estructura económica dentro de los sistemas sociales (OECD 1997, Millennium Assessment 2005).

Durante un largo periodo de tiempo no se catalogó como una actividad capaz de ocasionar impactos o efectos negativos sobre el ambiente, aunque en la actualidad se conoce que es susceptible de ocasionar graves daños en el entorno, los cuales pueden superar en magnitud a los provocados por diversos ámbitos industriales (LIFE03 2006).

Recientemente se ha presentado a la agricultura como un gran motor de transgresión de los llamados límites planetarios, indicándose que principalmente repercute sobre la integridad de la biosfera (pérdida de biodiversidad) y sobre los ciclos biogeoquímicos (principalmente de N y P; Campbell et al. 2017), así como sobre la introducción de entes novedosos (organismos modificados y sustancias tóxicas exógenas).

Una de las estrategias para aumentar la producción es la utilización continua de fertilizantes químicos y diversos tipos de plaguicidas destinados a prevenir, destruir o controlar plagas (Becerra y Bravo 2010). Estos productos son responsables de la denominada contaminación difusa (Turner et al. 2001, LIFE03 2006).

Los ecosistemas acuáticos aparecen como el destino final de la mayoría de estos contaminantes de origen antrópico (Amiard-Triquet 2015a, Del Arco 2015), que producen problemas de impacto ecológico relacionados con el descenso de la calidad de las aguas, generando alteraciones en las características y procesos ecológicos de los mismos (Parra et al. 2005, Guerrero et al. 2006).
Debido a la necesidad de evaluación del riesgo ambiental del uso de estas sustancias, en los últimos años se han incrementado notablemente los estudios sobre los efectos de la contaminación procedente de la agricultura en ecosistemas acuáticos (Nowell et al. 2014, Bendis y Relyea 2016, Barmentlo et al. 2018, Chará-Serna y Richardson 2018).

La evaluación puede llevarse a cabo a través de la información proporcionada por la determinación de parámetros físicos, químicos y biológicos, uso y detección de bioindicadores de contaminación y por la realización de bioensayos en laboratorio y en mesocosmos (Amiard-Triquet 2015b). Estos últimos proporcionan información de mayor utilidad a la hora de realizar la evaluación del riesgo ambiental de un determinado contaminante en comunidades acuáticas (Parra et al. 2005).

El fosfito de sodio actúa como fungicida selectivo y sistémico, beneficia al cuidado y control de cultivos y es categoría toxicológica III, de bajo impacto ambiental, poco peligrosa para el hombre, los animales y el ambiente (Velandia et al. 2012), presentando así una toxicidad muy baja para organismos no diana (Swiecki y Bernhardt 2013).

En el cuadro I se puede ver un resumen de los estudios publicados sobre fosfito sódico en organismos acuáticos.

A pesar de su baja toxicidad, las sales del ácido fosforoso (hidrogenofosfonatos y fosfonatos $=$ fosfitos) sufren una oxidación a iones fosfato, bien de carácter biológica (oxidación microbiana) o no biológica (Lovatt y Mikkelsen 2006, EFSA 2013). Por lo que el fosfito de sodio podría tener más repercusión por el enriquecimiento del medio acuático que por su efecto tóxico.

Una excesiva entrada de fósforo en los ecosistemas acuáticos tendría el potencial de causar eutrofización (Kim et al. 2013), que puede impactar 
CUADRO I. RESUMEN DE ESTUDIOS PUBLICADOS SOBRE FOSFITO DE SODIO Y SEMEJANTES.

\begin{tabular}{|c|c|c|c|c|c|}
\hline Artículo & Organismo & Producto & $\begin{array}{l}\text { Concentración } \\
\text { referente }\end{array}$ & $\begin{array}{l}\text { Diseño } \\
\text { experimental }\end{array}$ & $\begin{array}{l}\text { Observaciones en } \\
\text { estudio }\end{array}$ \\
\hline $\begin{array}{l}\text { (EFSA } \\
2013)\end{array}$ & D. magna & Disodium Phosphate & $\operatorname{EC} 50(61,26 \mathrm{mg} / \mathrm{L})$ & $\begin{array}{l}\text { Ensayo de toxicidad } \\
\text { aguda } 48 \mathrm{~h} \text { (static) }\end{array}$ & Mortalidad, EC50 \\
\hline $\begin{array}{l}\text { (EFSA } \\
2013)\end{array}$ & D. magna & Disodium Phosphate & $\mathrm{EC} 50(22,87 \mathrm{mg} / \mathrm{L})$ & $\begin{array}{l}\text { Ensayo de toxicidad } \\
\text { crónica } 21 \mathrm{~d} \\
\text { (semi-static) }\end{array}$ & Reproducción, NOEC \\
\hline $\begin{array}{l}\text { (EFSA } \\
2013)\end{array}$ & D. magna & $\begin{array}{l}\text { MILDICUT® }(25 \mathrm{~g} \\
\text { cyazofamid/L; } 250 \mathrm{~g} \\
\text { disodium phosphonate } / \mathrm{L})\end{array}$ & EC50 (21mg/L) & $\begin{array}{l}\text { Ensayo de toxicidad } \\
\text { aguda } 48 \mathrm{~h} \text { (static) }\end{array}$ & Mortalidad, EC50 \\
\hline $\begin{array}{l}\text { (Li et al. } \\
2015)\end{array}$ & D. magna & $\begin{array}{l}\text { Tris(1,3-dichloro-2- } \\
\text { propyl) phosphate } \\
\text { (TDCIPP) }\end{array}$ & $\begin{array}{l}\text { Máxima concentra- } \\
\text { ción reportada ( } 377 \\
\text { ng/L) }\end{array}$ & $\begin{array}{l}\text { Ensayo de } \\
\text { toxicidad crónica } \\
21 \mathrm{~d}(\text { semi-static) }\end{array}$ & $\begin{array}{l}\text { Crecimiento, } \\
\text { reproducción y } \\
\text { transcripción de genes }\end{array}$ \\
\hline $\begin{array}{l}\text { (Giraudo et al. } \\
\text { 2017) }\end{array}$ & D. magna & $\begin{array}{l}\text { Tris (2-butoxyethyl) } \\
\text { phosphate (TBOEP) }\end{array}$ & $\begin{array}{l}\text { Concentración } \\
\text { relevante en } \\
\text { sistemas }(10 \mu 1 / \mathrm{L})\end{array}$ & $\begin{array}{l}\text { Ensayo de toxicidad } \\
\text { crónica } 21 \mathrm{~d} \text { (semi- } \\
\text { static and during } 3 \\
\text { generations) }\end{array}$ & $\begin{array}{l}\text { Supervivencia, } \\
\text { reproducción y } \\
\text { crecimiento }\end{array}$ \\
\hline $\begin{array}{l}\text { (Douville et al. } \\
\text { 2016) }\end{array}$ & D. magna & $\begin{array}{l}\text { Tris (2-butoxyethyl) } \\
\text { phosphate (TBOEP) }\end{array}$ & LC50 (320mg/L) & $\begin{array}{l}\text { Ensayo de toxicidad } \\
\text { aguda } 48 \mathrm{~h} \text { (static) }\end{array}$ & $\begin{array}{l}\text { Mortalidad y efectos } \\
\text { subletales }\end{array}$ \\
\hline $\begin{array}{l}\text { (Douville et al. } \\
\text { 2016) }\end{array}$ & D. magna & $\begin{array}{l}\text { Tris (2-ethylhexyl) } \\
\text { Phosphate (TEHP) }\end{array}$ & LC50 (200mg/L) & $\begin{array}{l}\text { Ensayo de toxicidad } \\
\text { aguda } 48 \mathrm{~h} \text { (static) }\end{array}$ & $\begin{array}{l}\text { Mortalidad y efectos } \\
\text { subletales }\end{array}$ \\
\hline $\begin{array}{l}\text { (Douville et al. } \\
\text { 2016) }\end{array}$ & D. magna & $\begin{array}{l}\text { Bis (2-ethylhexyl) } \\
\text { phosphate (BEHP) }\end{array}$ & LC50 (180mg/L) & $\begin{array}{l}\text { Ensayo de toxicidad } \\
\text { aguda } 48 \mathrm{~h} \text { (static) }\end{array}$ & $\begin{array}{l}\text { Mortalidad y efectos } \\
\text { subletales }\end{array}$ \\
\hline
\end{tabular}

EC50 = concentración de efecto para el 50\% de la población, NOEC $=$ concentración de no efecto observado, LC50 = concentración letal mediana

y desestabilizar significativamente los mismos mediante la pérdida de hábitat, diversidad de especies y cambios en la estructura de la comunidad (Vitousek et al. 1997, Carpenter et al. 1998, LIFE03 2006, Jessen et al. 2015).

El crecimiento del fitoplancton, perifiton y plantas acuáticas (hidrófitas) se ve favorecido debido a una mayor abundancia de nutrientes, comenzando por el aumento de la biomasa y seguido de cambios en las especies dominantes y disminución de la diversidad (Mazzeo et al. 2002).

Por todo ello la hipótesis de este trabajo es que la contaminación de sistemas acuáticos con fosfito de sodio procedente de la agricultura podría ocasionar cambios en la estructura de la comunidad planctónica.

Se diseñó un experimento de larga duración (60 días) utilizando microcosmos con una comunidad planctónica que iba a ser expuesta a distintas concentraciones de fosfito de sodio. Para determinar las concentraciones se diseñó previamente un experimento crónico de toxicidad uniespecífico. En dicho experimento se expuso a Daphnia magna, cladócero utilizado en ensayos toxicológicos, durante 21 días para detectar efectos subletales bajo condiciones de alimentación óptimas y subóptimas.

Con el experimento de microcosmos se pretende determinar el efecto de la exposición del fungicida fosfito de sodio i) sobre la comunidad zooplanctónica mediante el análisis de la composición taxonómica y la abundancia de organismos durante el periodo de exposición experimental y ii) sobre la producción primaria mediante el análisis de la concentración de clorofila y el crecimiento de perifiton.

\section{MATERIAL Y MÉTODOS}

\section{Cultivo de Daphnia magna}

El cultivo de laboratorio de D. magna fue una población monoclonal criada a partir de neonatos de segunda generación. Los individuos se mantuvieron en un acuario de 15 litros a una temperatura de $20^{\circ} \mathrm{C}$, un fotoperiodo luz:oscuridad de 12:12 horas, con agua mineral (descrita más adelante) y una alimen- 
tación basada en una mezcla de algas Scenedesmus obliquus (Turpin) Kützing 1833 (Laboratorio de Ingeniería Química, Universidad de Jaén) y Cryptomonas pyrenoidifera Geitler 1922 (Instituto del Agua, Universidad de Granada) en una relación de al menos $1.5 \times 10^{6}$ células de algas/individuo.

Las algas anteriormente mencionadas se mantuvieron de forma rutinaria en medio de cultivo $3 \mathrm{~N}$ $\mathrm{BBM}+\mathrm{V}$ (receta de CCAP, Escocia, basada en Bold's basal medium BBM) con $\mathrm{pH}$ corregido a 8.3-8.5 con hidróxido de sodio $(\mathrm{NaOH})$ antes de esterilización mediante autoclave.

\section{Ensayos de toxicidad crónica con D. magna}

Para la realización de los dos ensayos de toxicidad crónica se ha seguido el protocolo de la prueba de reproducción para D. magna (OECD 2012). Se utilizó fosfito de sodio dibásico pentahidratado de la marca ${ }^{\circledR}$ Sigma-Aldrich para la preparación de una solución madre a una concentración final de $250 \mathrm{mg} / \mathrm{L}$ de fosfito de sodio.

Para llevar a cabo los ensayos crónicos uniespecíficos se prepararon tres concentraciones subletales: 5 mg/L (bajo, B), 10 mg/L (medio, M), 25 mg/L (alto, A) con base en la $\mathrm{LC}_{50}$ publicada por varios autores (Cuadro I). Además de estas tres concentraciones de tóxico, también se expuso en el ensayo testigo (T), libre de concentración de fungicida.

En cada experimento se prepararon cuatro réplicas para cada concentración o tratamiento en las que se expuso a los organismos de forma individual en frascos de vidrio de $60 \mathrm{~mL}$ de capacidad, con $50 \mathrm{~mL}$ de medio (agua mineral suplementada con vitaminas según tipo de experiencia, ver más adelante), con las siguientes características: $\mathrm{pH}=8.28$, temperatura $=$ $20^{\circ} \mathrm{C}$, dureza $=219.96 \mathrm{mg} / \mathrm{L} \mathrm{CaCO}_{3}$ y conductividad eléctrica $=0.48 \mathrm{mS} / \mathrm{cm}$ ). Se utilizaron 16 neonatos $(<24 \mathrm{~h})$ procedentes de una línea monoclonal.

Se planificaron dos experiencias, en una de ellas los organismos eran mantenidos a unas condiciones de cultivo subóptimas (con concentración de algas menor a $1.5 \times 10^{6}$ células por dafnia/día y sin añadir vitaminas al medio). Esta experiencia simuló una situación probablemente más parecida a la que tiene lugar en el medio natural donde la disponibilidad de recursos es variable (disponibilidad, calidad, competencia).

En la segunda experiencia se mantuvieron condiciones óptimas con alimentación ad libitum y disponibilidad mayor de vitaminas siguiendo el ejemplo de la experiencia de Díaz-Báez et al. (2004). Se añadió así a cada uno de los frascos, $5 \mu \mathrm{L}$ de tiamina, $1 \mu \mathrm{L}$ de vitamina B12, $0.5 \mu \mathrm{L}$ de biotina y $1 \mu \mathrm{L}$ de selenito de sodio (Elendt y Bias 1990), una vez por semana.
A partir del día 7 se fueron retirando los neonatos que iban emergiendo empleando pipetas Pasteur. Estos neonatos fueron aislados en función de su tratamiento de origen y mantenidos en condiciones óptimas, para así poder estudiar los efectos del producto tóxico en su supervivencia.

Finalizados 21 días de experiencia se realizó el recuento total de la reproducción en cada uno de los vasos experimentales, además se analizó el tamaño final de estos, para así poder evaluar la existencia o no de diferencias en crecimiento.

Para la estimación de la longitud, se midió el caparazón, desde la región anterior del rostro hasta el final de la espina apical. Los individuos fueron dispuestos en la lupa (Leica MZ12) que presenta una cámara Leica EC3 acoplada. Una vez tomada la imagen y almacenada en el ordenador se midieron todos los sujetos experimentales con el programa de análisis de imagen "Imagen J".

\section{Establecimiento de microcosmos}

En total 12 microcosmos (recipientes de polipropileno, con forma de cono truncado, con una capacidad total de $50 \mathrm{~L}$ y con las siguientes dimensiones: radio de la base grande $=22.25 \mathrm{~cm}$, radio de la base pequeña $=16.35 \mathrm{~cm}$ y altura $=42.40 \mathrm{~cm}$ ) se llenaron con $40 \mathrm{~L}$ de agua de grifo y se situaron al aire libre en las inmediaciones del humedal experimental de la Universidad de Jaén (Humexpuja) (Jaén, Andalucía, España; $37^{\circ} 47.2626919298628^{\prime}$ N, $3^{\circ} 46.71190649271011^{\prime} \mathrm{W}$, clima mediterráneo).

Cada uno de ellos se cubrió con una malla metálica para evitar la entrada de hojarasca y otros organismos. Para la completa eliminación del cloro se llenaron 48 horas antes de añadir los organismos planctónicos que formarían la comunidad.

Con la finalidad de permitir la estabilización de los microcosmos, el inicio del experimento se produjo cinco semanas después de la inoculación de la comunidad planctónica.

La captura de organismos zooplanctónicos del Humexpuja se realizó en diferentes puntos de mismo con una red de plancton de $43 \mu \mathrm{m}$ de luz de malla, en total se hicieron 35 lances. Tras cada lance se procedió a lavar la red de plancton con un spray a presión, que contenía agua del Humexpuja, utilizando la mínima cantidad posible para lograr la concentración de dichos organismos. El agua de cada uno de los lavados se recogió en un mismo recipiente y a continuación se procedió a la mezcla moderada de forma manual hasta la completa homogeneización de misma.

Posteriormente se distribuyeron $400 \mathrm{~mL}$ de forma equitativa a cada uno de los microcosmos. Además, 
también de forma homogénea, se añadieron $1.3 \mathrm{~L}$ de agua del Humexpuja a cada uno de ellos para asegurar el crecimiento de la comunidad fitoplanctónica.

\section{Experimento de exposición en microcosmos}

Las concentraciones seleccionadas después de analizados los resultados de la experiencia con $D$. magna fueron: $5 \mathrm{mg} / \mathrm{L}(\mathrm{B})$ y $10 \mathrm{mg} / \mathrm{L}(\mathrm{M})$, además de un tratamiento testigo (T). El criterio de selección se basó en la respuesta de los neonatos en el ensayo 2 bajo condiciones subóptimas que representan un escenario más realista de un medio donde la disponibilidad de recursos es limitada y sujeta a interacciones con otras especies, así como a cambios ambientales.

Cada uno de los tratamientos contó con cuatro réplicas. La adjudicación de los diferentes tratamientos a los microcosmos se realizó de forma aleatoria. Los tratamientos con fosfito de sodio se añadieron el primer día del experimento que tuvo una duración total de ocho semanas con muestreos semanales de todos los indicadores que se detallan a continuación.

Las variables físicas y químicas de los microcosmos fueron medidas semanalmente in situ utilizando una sonda multiparamétrica de campo YSI-556MPS. Las variables determinadas fueron: temperatura $\left({ }^{\circ} \mathrm{C}\right)$, conductividad $\left(\mathrm{mS} / \mathrm{cm}^{3}\right)$, total de sólidos disueltos (TSD g/L), oxígeno disuelto $(\mathrm{mg} / \mathrm{L})$ y $\mathrm{pH}$.

La comunidad zooplanctónica se analizó en cada uno de los microcosmos mediante la toma de una muestra de agua de $2 \mathrm{~L}$ que se filtró ( $41 \mu \mathrm{m}$ tamaño de malla) y fijó con formaldehido (4\%), mientras que el agua filtrada se devolvió a su respectivo microcosmo. A continuación, se procedió a contabilizar e identificar los grupos de zooplancton con una lupa de laboratorio Leica 2000.

La comunidad fitoplanctónica se evaluó mediante fluorescencia, este es un dato directo de emisión de clorofila, por lo que cambios en sus valores son de gran utilidad para detectar procesos de estrés ambiental (González et al. 2008, Ceacero et al. 2012). Los valores de fluorescencia en partes por billón (ppb) se obtuvieron utilizando el fluorímetro de mano Aquaflor de Turner Designs.

Para estimar el crecimiento del perifiton durante el periodo experimental se introdujeron tres portaobjetos de cristal, como soporte artificial, en cada uno de los microcosmos. Una vez finalizado el experimento se procedió a retirar los portaobjetos de cada uno de los microcosmos, con cuidado de no eliminar parte de la muestra. Con la finalidad de determinar el peso seco del perifiton crecido durante el periodo experimental, los portaobjetos se introdujeron en estufa a una temperatura de $50{ }^{\circ} \mathrm{C}$ durante 24 h para así garantizar su secado.

Una vez transcurrido ese tiempo, las muestras se dejaron enfriar en un desecador que contenía gel de sílice y cuando alcanzaron la temperatura ambiente se procedió a determinar el peso del perifiton. Para ello, con ayuda de unas pinzas de laboratorio, se calcula el peso de las cápsulas de pesaje (previamente fueron secadas y enfriadas de igual manera que los portaobjetos). A continuación se procede al raspado del respectivo porta con ayuda de otro porta limpio sobre la cápsula, y se pesa la cápsula y el perifiton. De tal manera que el peso del perifiton se obtiene restando al peso de la cápsula con perifiton del peso de la cápsula vacía.

\section{Análisis estadísticos}

En el experimento crónico con D. magna los resultados de supervivencia, tamaño y reproducción fueron analizados estadísticamente con el programa SPSS, realizando comparación de valores medios mediante un análisis de la varianza de un factor y un análisis de Tukey al final del periodo experimental.

En el experimento con microcosmos, los datos de parámetros físicos y químicos, zooplancton, fitoplancton y perifiton fueron analizados estadísticamente con el programa SPSS. Mediante la prueba $\mathrm{H}$ de Kruskal-Wallis se analizó la posible existencia de diferencias significativas entre tratamientos en la semana ocho.

Se utilizó un análisis de la varianza de medidas repetidas y la prueba post hoc de Tukey para analizar si existían diferencias significativas entre tratamientos a lo largo de todo el periodo experimental.

Tanto para los análisis del ensayo crónico como de microcosmos se comprobó si los datos cumplían con la normalidad mediante las pruebas de normalidad Kolmogorov-Smimov y Shapiro-Wilk. En caso de ser necesario, los datos fueron transformados (con el logaritmo o la raíz cuadrada dependiendo del tipo de dato).

Además, en la evaluación de la comunidad zooplanctónica en el experimento de microcosmos, se utilizó el programa CANOCO (5.0) para realizar un análisis de la respuesta principal (PRC, por sus siglas en inglés) de la comunidad. Este permite observar de manera conjunta la respuesta de todos los taxones que componen la comunidad en un único diagrama.

Dicho diagrama presenta las semanas de muestreo en el eje x y la respuesta principal de la comunidad en el eje y, mostrando las desviaciones con el tiempo de los tratamientos frente a los testigos (Moser et al. 2007, Zafar et al. 2012, Del Arco Ochoa 2015). 
Un segundo gráfico (1-D plot), complementario del anterior, representa el peso del taxón, el cual indica el grado de afinidad de los diferentes taxones con la respuesta general mostrada por la PRC.

En función del valor del peso de los taxones, los cambios que sufran pueden estar directamente, indirectamente o no correlacionados con la tendencia de la respuesta principal. Si el peso del taxón presenta valores positivos esto indica correlaciones positivas con la respuesta principal de la comunidad, si el valor es negativo la correlación es negativa y si el valor es cercano a cero significa que no hay respuesta o que es muy diferente a la respuesta principal (Van den Brink y Ter Braak 1999, Zafar et al. 2012).

Además, el programa permite evaluar la significancia estadística de los efectos del tratamiento en la composición de especies de los microcosmos mediante el método de permutación Monte Carlo, el cual muestra el cociente $\mathrm{F}$ y el valor $\mathrm{p}$ para la significancia del primer eje canónico del PRC (Moser et al. 2007, Zafar et al. 2012). Para el tratamiento de datos, estos fueron transformados mediante $\ln (2 \mathrm{x}+1)$ (Zafar et al. 2012).

\section{RESULTADOS}

\section{Experimento crónico con D. magna}

El cuadro II resume los resultados obtenidos en supervivencia, reproducción y tamaño final de los adultos en los dos ensayos, bajo condiciones subóptimas y óptimas. Bajo condiciones subóptimas (ensayo 1) no se consiguió obtener neonatos en los tratamientos $\mathrm{M}$ y A.

En cuanto al C y B mostraron valores muy bajos de reproducción, sin diferencias significativas en número de neonatos $\left(\mathrm{F}_{(3,6)}=0.451 ; \mathrm{p}=0.527\right)$. En cuanto al tamaño hay diferencias significativas entre tratamiento $\left(\mathrm{F}_{(3,10)}=8.429 ; \mathrm{p}=0.004\right)$. Siendo el C el que mayor tamaño medio mantiene al final del experimento.

Bajo condiciones óptimas (ensayo 2), no hay diferencias significativas en número de neonatos totales producidos $\left(\mathrm{F}_{(3,12)}=2.120 ; \mathrm{p}=0.151\right)$, ni en el tamaño final alcanzado $\left(\mathrm{F}_{(3,12)}=2.182 ; \mathrm{p}=0.143\right)$.

\section{Experimento de microcosmos}

Teniendo en cuenta los valores promedio de los parámetros físicos y químicos $\mathrm{y}$ sus respectivas desviaciones estándar, podemos observar que a lo largo de todo el periodo experimental dichos parámetros presentan una evolución similar, existiendo diferencias significativas a lo largo del tiempo (oxígeno: $\mathrm{F}=33.13 ; \mathrm{p}<0.0001$; temperatura: $\mathrm{F}=19.79$; $\mathrm{p}<0.0001$; conductividad: $\mathrm{F}=27.33 ; \mathrm{p}<0.0001$; $\mathrm{pH}: \mathrm{F}=33,131 ; \mathrm{p}<0,000 ; \mathrm{TSD}: \mathrm{F}=27.84$; $\mathrm{p}<0,0001$ ), pero no entre tratamientos, a excepción del $\mathrm{pH}$ en la semana 6 , donde el $\mathrm{C}$ mostró valores más bajos que $\mathrm{B}$ y $\mathrm{M}$ (oxígeno: $\mathrm{F}_{(2,9)}=0.797 ; \mathrm{p}=$ 0.480 ; temperatura: $\mathrm{F}_{(2,9)}=1.94 ; \mathrm{p}=0.199$; conductividad: $\mathrm{F}_{(2,9)}=1.23 ; \mathrm{p}=0.336 ; \mathrm{pH}: \mathrm{F}_{(2,9)}=8 ; \mathrm{p}=$ 0.010 ; TSD: $\left.\mathrm{F}_{(2,9)}=1.22 ; \mathrm{p}=0.339\right),($ Cuadro III

CUADRO II. PORCENTAJE EN SUPERVIVENCIA DE ADULTOS Y NEONATOS JUNTO A VALORES PROMEDIO DE INDICADORES DE REPRODUCCIÓN Y TAMAÑO FINAL DEL ADULTO EN LOS DIFERENTES TRATAMIENTOS

\begin{tabular}{lcccccc}
\hline Ensayo 1 & \multicolumn{7}{c}{ Condiciones subóptimas de alimentación } \\
\hline Parámetro/ tratam. & $\begin{array}{c}\text { Superv. } \\
(\%)\end{array}$ & Neonatos & $\begin{array}{c}\text { Tamaño } \\
\text { camada }\end{array}$ & $\begin{array}{c}\text { Núm. de } \\
\text { camadas }\end{array}$ & $\begin{array}{c}\text { Tiempo } \\
1^{\mathrm{a}} \text { puesta (días) }\end{array}$ & $\begin{array}{c}\text { Longitud } \\
(\mathrm{mm})\end{array}$ \\
\hline Testigo & 100 & $4.3 \pm 3.9$ & $4.3 \pm 3.6$ & $1.0 \pm 0.8$ & $13.5 \pm 0.6$ & $2.6 \pm 0.2$ \\
Bajo & 100 & $2.5 \pm 3.0$ & $5.0 \pm 1.4$ & $0.5 \pm 0.6$ & $13.5 \pm 0.7$ & $2.3 \pm 0.3$ \\
Medio & 75 & - & - & - & - & $0.3 \pm 0.1$ \\
Alto & 75 & - & - & - & - & $0.3 \pm 0.1$ \\
\hline Ensayo 2 & \multicolumn{7}{c}{ Condiciones óptimas de alimentación } & \\
\hline Testigo & 100 & $103.8 \pm 32.1$ & $25.9 \pm 11.3$ & $4.0 \pm 0.8$ & $11.0 \pm 2.2$ & $4.5 \pm 0.2$ \\
Bajo & 100 & $89.5 \pm 17.7$ & $19.9 \pm 8.3$ & $4.5 \pm 0.5$ & $9.5 \pm 0.6$ & $4.1 \pm 0.2$ \\
Medio & 100 & $64.7 \pm 14.8$ & $17.5 \pm 9.1$ & $4.5 \pm 0.6$ & $10.0 \pm 0.8$ & $4.4 \pm 0.2$ \\
Alto & 100 & $63.3 \pm 24.4$ & $18.7 \pm 9.3$ & $3.8 \pm 0.5$ & $1.0 \pm 0.0$ & $4.0 \pm 0.5$ \\
\hline
\end{tabular}

Testigo, sin tóxico (0 mg/L); concentración baja $5 \mathrm{mg} / \mathrm{L}$ (Bajo); concentración media (10 mg/L Medio); concentración alta $(25 \mathrm{mg} / \mathrm{L}$ Alto). 
CUADRO III. VALORES PROMEDIO DE LOS PARÁMETROS FÍSICOS Y QUÍMICOS CON SUS RESPECTIVAS DESVIACIONES ESTÁNDAR EN LOS TRES TRATAMIENTOS A LO LARGO DEL PERIODO EXPERIMENTAL: A) SEMANAS 0,1 Y 2; B) SEMANAS 3, 4 Y 5; C) SEMANAS 6, 7 Y 8.

\begin{tabular}{|c|c|c|c|c|c|c|c|c|c|}
\hline \multicolumn{10}{|l|}{ A) } \\
\hline \multirow{2}{*}{$\begin{array}{l}\text { Semana } \\
\text { Tratamiento/ } \\
\text { parámetro }\end{array}$} & \multicolumn{3}{|c|}{0} & \multicolumn{3}{|c|}{1} & \multicolumn{3}{|c|}{2} \\
\hline & $\mathrm{T}$ & $\mathrm{B}$ & M & $\mathrm{T}$ & $\mathrm{B}$ & $\mathrm{M}$ & $\mathrm{T}$ & $\mathrm{B}$ & M \\
\hline Tem. $\left({ }^{\circ} \mathrm{C}\right)$ & $13.24 \pm 0.13$ & $13.25 \pm 0.16$ & $13.21 \pm 0.15$ & $14.56 \pm 0.35$ & $14.33 \pm 0.20$ & $14.69 \pm 0.94$ & $14.98 \pm 0.12$ & $14.76 \pm 0.17$ & $14.99 \pm 0.35$ \\
\hline $\mathrm{OD}(\mathrm{mg} / \mathrm{L})$ & $8.83 \pm 0.32$ & $8.76 \pm 0.31$ & $8.77 \pm 0.21$ & $8.42 \pm 0.53$ & $8.10 \pm 0.36$ & $8.82 \pm 0.32$ & $7.62 \pm 0.47$ & $7.26 \pm 0.61$ & $7.68 \pm 0.31$ \\
\hline $\mathrm{pH}$ & $8.31 \pm 0.02$ & $8.35 \pm 0.03$ & $8.31 \pm 0.02$ & $8.43 \pm 0.06$ & $8.41 \pm 0.03$ & $8.43 \pm 0.09$ & $8.43 \pm 0.13$ & $8.38 \pm 0.11$ & $8.46 \pm 0.15$ \\
\hline Cond. (mS/cm $\left.{ }^{3}\right)$ & $0.65 \pm 0.06$ & $0.61 \pm 0.03$ & $0.67 \pm 0.10$ & $0.62 \pm 0.06$ & $0.58 \pm 0.04$ & $0.65 \pm 0.10$ & $0.64 \pm 0.07$ & $0.60 \pm 0.05$ & $0.67 \pm 0.10$ \\
\hline TSD (g/L) & $0.42 \pm 0.04$ & $0.39 \pm 0.02$ & $0.43 \pm 0.07$ & $0.40 \pm 0.04$ & $0.38 \pm 0.03$ & $0.43 \pm 0.07$ & $0.41 \pm 0.04$ & $0.39 \pm 0.03$ & $0.44 \pm 0.07$ \\
\hline \multicolumn{10}{|l|}{ B) } \\
\hline Semana & & 3 & & & 4 & & & 5 & \\
\hline Tem. $\left({ }^{\circ} \mathrm{C}\right)$ & $16.19 \pm 0.33$ & $16.27 \pm 0.16$ & $16.97 \pm 0.66$ & $16.87 \pm 0.24$ & $16.79 \pm 0.19$ & $16.84 \pm 0.09$ & $12.07 \pm 0.10$ & $12.30 \pm 0.23$ & $12.62 \pm 0.19$ \\
\hline $\mathrm{OD}(\mathrm{mg} / \mathrm{L})$ & $7.03 \pm 0.22$ & $7.34 \pm 0.17$ & $7.43 \pm 0.49$ & $7.13 \pm 0.42$ & $7.60 \pm 0.47$ & $6.93 \pm 0.71$ & $7.71 \pm 0.85$ & $8.79 \pm 0.42$ & $7.94 \pm 1.54$ \\
\hline $\mathrm{pH}$ & $8.48 \pm 0.05$ & $8.49 \pm 0.04$ & $8.52 \pm 0.01$ & $8.43 \pm 0.06$ & $8.51 \pm 0.04$ & $8.50 \pm 0.05$ & $8.38 \pm 0.07$ & $8.63 \pm 0.09$ & $8.83 \pm 0.07$ \\
\hline Cond. $\left(\mathrm{mS} / \mathrm{cm}^{3)}\right.$ & $0.65 \pm 0.08$ & $0.61 \pm 0.06$ & $0.68 \pm 0.11$ & $0.70 \pm 0.08$ & $0.65 \pm 0.06$ & $0.72 \pm 0.11$ & $0.66 \pm 0.11$ & $0.55 \pm 0.07$ & $0.66 \pm 0.13$ \\
\hline $\mathrm{TSD}(\mathrm{g} / \mathrm{L})$ & $0.42 \pm 0.05$ & $0.40 \pm 0.04$ & $0.44 \pm 0.07$ & $0.46 \pm 0.05$ & $0.42 \pm 0.04$ & $0.47 \pm 0.07$ & $0.43 \pm 0.07$ & $0.35 \pm 0.04$ & $0.43 \pm 0.09$ \\
\hline \multicolumn{10}{|l|}{ C) } \\
\hline Semana & & 6 & & & 7 & & & 8 & \\
\hline Tem. $\left({ }^{\circ} \mathrm{C}\right)$ & $20.31 \pm 0.29$ & $20.77 \pm 0.43$ & $21.68 \pm 0.68$ & $20.31 \pm 0.29$ & $20.77 \pm 0.43$ & $21.68 \pm 0.68$ & $20.31 \pm 0.29$ & $20.77 \pm 0.43$ & $21.68 \pm 0.68$ \\
\hline $\mathrm{OD}(\mathrm{mg} / \mathrm{L})$ & $6.66 \pm 0.82$ & $6.74 \pm 0.57$ & $6.76 \pm 0.66$ & $6.66 \pm 0.82$ & $6.74 \pm 0.57$ & $6.76 \pm 0.66$ & $6.66 \pm 0.82$ & $6.74 \pm 0.57$ & $6.76 \pm 0.66$ \\
\hline $\mathrm{pH}$ & $8.49 \pm 0.08$ & $8.61 \pm 0.08$ & $8.63 \pm 0.07$ & $8.49 \pm 0.08$ & $8.61 \pm 0.08$ & $8.63 \pm 0.07$ & $8.49 \pm 0.08$ & $8.61 \pm 0.08$ & $8.63 \pm 0.07$ \\
\hline Cond. (mS/cm ${ }^{3)}$ & $0.69 \pm 0.11$ & $0.57 \pm 0.07$ & $0.70 \pm 0.14$ & $0.69 \pm 0.11$ & $0.57 \pm 0.07$ & $0.70 \pm 0.14$ & $0.69 \pm 0.11$ & $0.57 \pm 0.07$ & $0.70 \pm 0.14$ \\
\hline $\mathrm{TSD}(\mathrm{g} / \mathrm{L})$ & $0.45 \pm 0.07$ & $0.37 \pm 0.05$ & $0.45 \pm 0.09$ & $0.45 \pm 0.07$ & $0.37 \pm 0.05$ & $0.45 \pm 0.09$ & $0.45 \pm 0.07$ & $0.37 \pm 0.05$ & $0.45 \pm 0.09$ \\
\hline
\end{tabular}

Testigo, sin tóxico $(0 \mathrm{mg} / \mathrm{L}, \mathrm{T})$, concentración baja $(5 \mathrm{mg} / \mathrm{L}, \mathrm{B})$, concentración media $(10 \mathrm{mg} / \mathrm{L}, \mathrm{M})$. Tem. $=$ temperatura, $\mathrm{OD}=$ oxígeno disuelto, Cond. $=$ conductividad, $\mathrm{TSD}=$ total de sólidos disueltos

a, b y c). Por tanto, las condiciones experimentales, aparte de la exposición al tóxico, son similares en todos los tratamientos, salvo en la semana 6 respecto al $\mathrm{pH}$.

En lo referente a la temperatura de los microcosmos, podemos ver que a lo largo de todo el periodo experimental este parámetro muestra un incremento, a excepción de las semanas 5 y 7, lo que concuerda con las variaciones de la temperatura ambiental registradas en la estación meteorológica de Jaén de la red de estaciones agroclimáticas de la Junta de Andalucía (2016).

Tras evaluar e identificar los grupos taxonómicos de las muestras de zooplancton, estos fueron clasificados en los siguientes ocho niveles taxonómicos prácticos (TPL, por sus siglas en inglés): nauplios, Metacyclops minutus, Daphnia sp., Alona sp., ostrácodos, Lecane luna, Lecane sp. y Hexarthra sp.

En la figura 1 se puede observar la evolución semanal de la abundancia total de organismos zoo- planctónicos en cada uno de los tratamientos a lo largo del experimento.

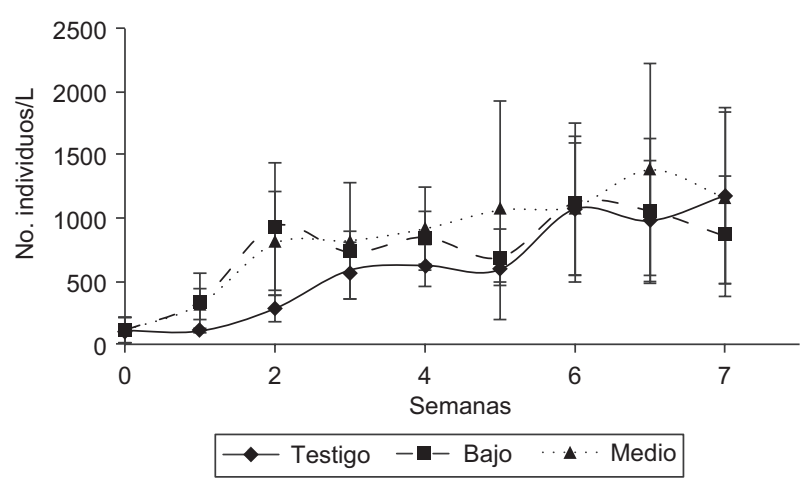

Fig. 1. Evolución semanal de la abundancia total de organismos zooplanctónicos en cada uno de los tratamientos a lo largo del experimento. Testigo, sin tóxico $(0 \mathrm{mg} / \mathrm{L})$, concentración baja ( $5 \mathrm{mg} / \mathrm{L}$, Bajo), concentración media ( 10 $\mathrm{mg} / \mathrm{L}$, Medio). Las barras de error indican la desviación estándar 
Con la finalidad de determinar si existían diferencias significativas respecto a los valores de abundancia total de zooplancton entre los tres tratamientos (C, B y M) se llevó a cabo la prueba Kruskal-Wallis.

El análisis estadístico de los resultados reveló que no existen diferencias significativas entre tratamientos a lo largo del periodo experimental a excepción de la semana $1\left(\mathrm{X}^{2}=7.423\right.$; g.l. $\left.=2 ; \mathrm{p}=0.024\right)$.

Inicialmente, las comunidades zooplanctónicas son poco diversas y están formadas casi en su totalidad por nauplios. Con el paso del tiempo empiezan a aparecer otros organismos zooplanctónicos, cuya abundancia también aumenta. De forma que, en las últimas semanas del experimento se observa un incremento de la riqueza de organismos zooplanctónicos que forman las comunidades de los microcosmos y una mayor similitud en la abundancia de cada uno de los TPL.

Sin embargo, cabe destacar que el grupo zooplanctónico más abundante durante todo el experimento han sido los nauplios. En el caso de B y M ambas comunidades presentan siete TPL, mientras que la de C posee ocho TPL, siendo así el único tratamiento en el cual se observa el rotífero Hexarthra sp.

El segundo TPL con el mayor rango de abundancia tanto en $\mathrm{B}$ como en $\mathrm{M}$ son los ostrácodos, a diferencia del $\mathrm{C}$ en donde lo es el cladócero Alona sp. seguido a continuación por los ostrácodos.

A partir de los datos biológicos recogidos la última semana del experimento, se construyeron las curvas de rango-abundancia de cada uno de los tratamientos (Fig. 2).

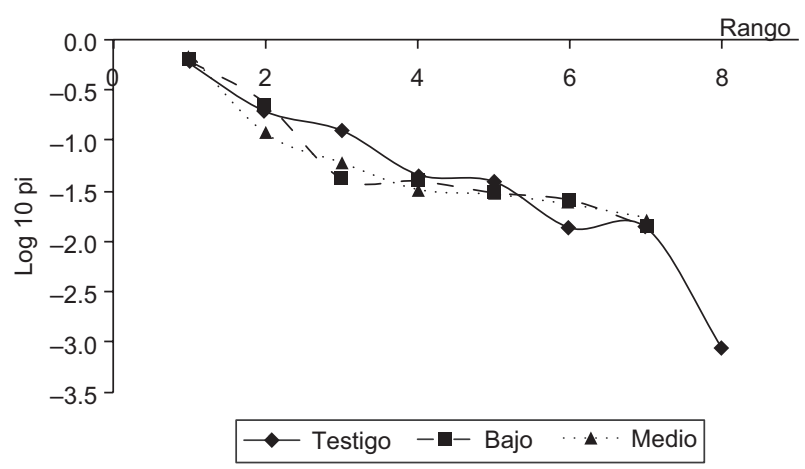

Fig. 2. Curvas de rango-abundancia de las comunidades que se han establecido en cada uno de los tratamientos al final del periodo experimental. Testigo, sin tóxico $(0 \mathrm{mg} / \mathrm{L})$; concentración baja $5 \mathrm{mg} / \mathrm{L}$ (Bajo); concentración media (10 mg/L Medio)

Teniendo en cuenta la pendiente de las curvas de rango-abundancia de los tres tratamientos $(\mathrm{C}, \mathrm{B}$ y $\mathrm{M})$ se puede deducir que son muy similares. Además, no hay diferencias significativas en el índice de diversidad de las comunidades al final del experimento (Fig. 3; $\left.\mathrm{F}_{(2,9)}=0.042 ; \mathrm{p}=0.960\right)$.

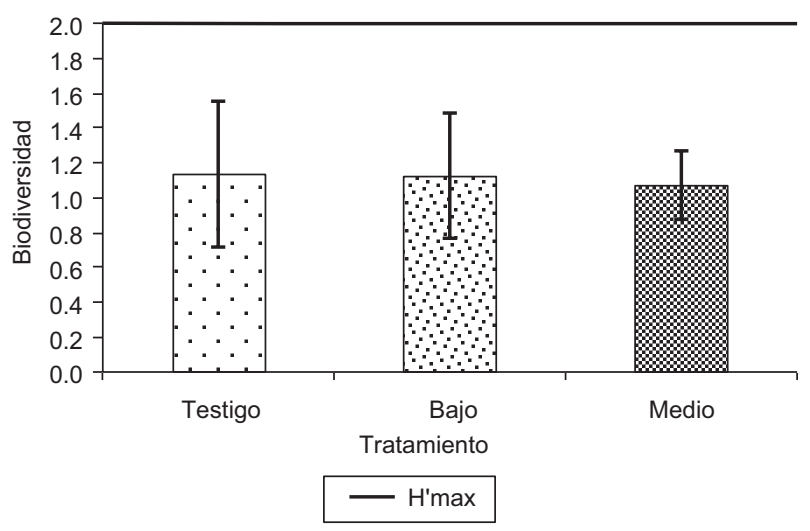

Fig. 3. Biodiversidad de las comunidades que se han establecido al final del experimento en los tres tratamientos. H'max = diversidad máxima si la distribución fuese equitativa en la comunidad. Testigo, sin tóxico $(0 \mathrm{mg} / \mathrm{L})$, concentración baja ( $5 \mathrm{mg} / \mathrm{L}$, Bajo), concentración media (10 $\mathrm{mg} / \mathrm{L}$, Medio). Las barras de error indican la desviación estándar

Dado que en ningún caso la comunidad zooplanctónica presenta un valor del índice de ShannonWiener máximo (H'max), en ninguno de los tratamientos la comunidad presentó una distribución de abundancias equitativa.

El diagrama PRC (Fig. 4) muestra la respuesta integrada de la estructura de la comunidad zooplanctónica debido a los tratamientos a lo largo del experimento. Se observa que tras la aplicación del fosfito de sodio dibásico pentahidratado hasta aproximadamente la segunda semana, la respuesta de las comunidades zooplanctónicas a los tratamientos B y $M$ presenta unas pequeñas desviaciones respecto a $\mathrm{C}$, siendo mayor la de $\mathrm{B}$.

Desde la semana dos hasta la semana ocho dichas desviaciones adquieren mayor relevancia, y durante este periodo de tiempo la desviación de $\mathrm{M}$ es mayor que la de B, presentando ambos una tendencia similar.

El único TPL cuyo peso presenta valor positivo es Hexarthra sp., por lo que sería el único cuya abundancia disminuirá tras el tratamiento con el fosfonato. Sin embargo, hay que tener en cuenta que como su valor se encuentra dentro del rango +0.5 y - 0.5 puede manifestar tanto una respuesta débil como una respuesta que no guarde relación con la mostrada en el diagrama. 


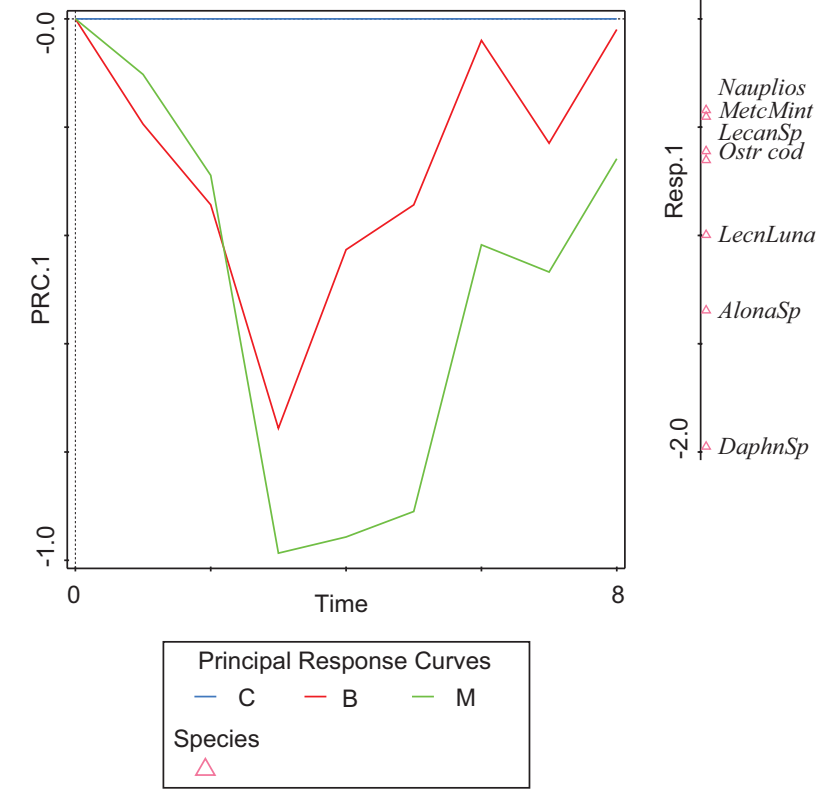

Fig. 4. La respuesta general de la estructura de la comunidad zooplanctónica y los cambios de abundancia de los niveles taxonómicos prácticos (TPL, por sus siglas en inglés) debidos al tratamiento: Testigo, sin tóxico $(0$ $\mathrm{mg} / \mathrm{L})$, concentración baja $(5 \mathrm{mg} / \mathrm{L}, \mathrm{B})$, concentración media $(10 \mathrm{mg} / \mathrm{L}, \mathrm{M})$ a lo largo del experimento están representados en el diagrama PRC (curvas de respuesta principal, por sus siglas en inglés). El peso de dichos TPL en la respuesta general de la comunidad está representado en el gráfico 1-D plot

El resto de taxones presentaron valores negativos, correspondiendo a Daphnia sp. el valor de peso negativo más alto seguido de Alona sp., que serán los TPL que presenten los mayores incrementos en abundancia debido al tratamiento.

De acuerdo con los resultados del método de permutación Monte Carlo $(\mathrm{F}=14 ; \mathrm{p}=0.07)$ no existen diferencias significativas entre tratamientos en lo que se refiere a la abundancia y a la estructura de la comunidad, aunque cabe destacar que dichos resultados se encuentran en el límite de la significancia.

Por tanto, aunque el agroquímico parece ocasionar variaciones en la comunidad zooplanctónica respecto al testigo $(\mathrm{C})$, estas no son significativas.

Los cambios en la concentración de clorofila estimada mediante las medidas de fluorescencia (Fig. 5) muestran tendencias diversas entre los tratamientos y los testigos.

El análisis estadístico de los resultados puso de manifiesto la existencia de diferencias significativas

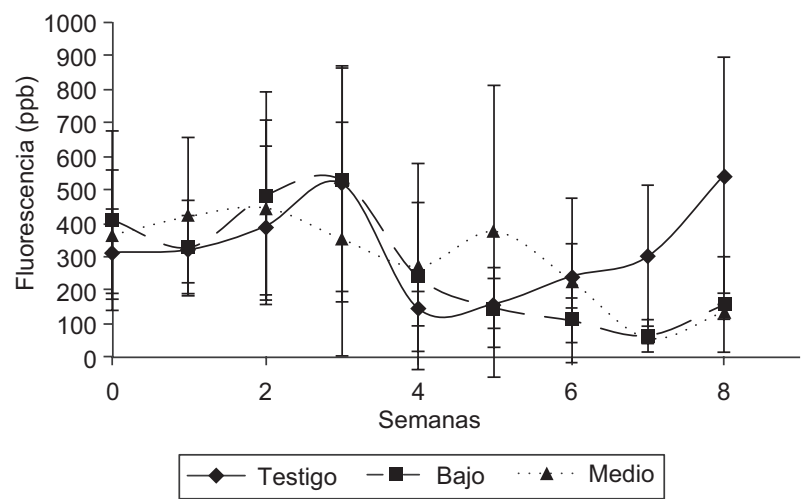

Fig. 5. Variación semanal de los valores de fluorescencia en cada uno de los tratamientos durante el periodo experimental. Testigo, sin tóxico $(0 \mathrm{mg} / \mathrm{L})$; concentración baja $(5 \mathrm{mg} / \mathrm{L}$, Bajo); concentración media (10 mg/L, Medio). Las barras de error indican la desviación estándar

en relación al tiempo $\left.\left(\mathrm{F}_{(8)}\right)=7.205 ; \mathrm{p}<0.001\right)$, pero no en relación al tratamiento $\left(\mathrm{F}_{(8,16)}=1.512 ; \mathrm{p}=\right.$ 0.119 ).

En las pruebas de los efectos intersujetos se ratifica la no existencia de diferencias significativas de los valores de fluorescencia debido a los tratamientos $(\mathrm{F}=0.841 ; \mathrm{p}=0.463)$.

Con respecto al perifiton, la figura 6 presenta las medias del crecimiento en función del tratamiento. El análisis estadístico de los resultados puso de manifiesto la existencia de diferencias significativas respecto al crecimiento del perifiton entre tratamientos $(\mathrm{F}=5.068 ; \mathrm{p}=0.012)$. El análisis mediante la prueba post hoc de Tukey muestra que el testigo (T) no presenta diferencias significativas respecto a $B(p$ $=0.654)$ y $\mathrm{M}(\mathrm{p}=0.085)$, pero sí existen diferencias significativas entre B y M $(\mathrm{p}=0.011)$.

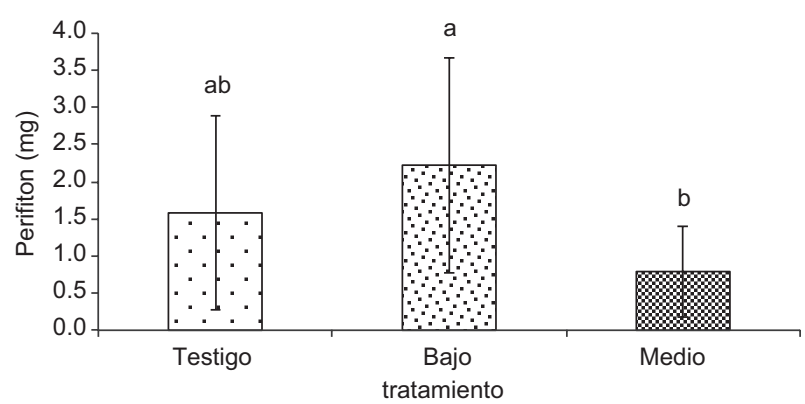

Fig. 6. Valor de biomasa medio (mg) del perifiton en cada tratamiento al final del periodo experimental. Testigo, sin tóxico $(0 \mathrm{mg} / \mathrm{L})$; concentración baja $(5 \mathrm{mg} / \mathrm{L}$, Bajo); concentración media (10 mg/L, Medio). Las barras de error indican la desviación estándar, letras diferentes indican diferencias significativas entre grupos 


\section{DISCUSIÓN}

Los resultados obtenidos en el experimento uniespecífico han mostrado la repercusión de las condiciones de experimentación y ambientales en la capacidad de respuesta de un individuo o una población frente a un tóxico. D. magna ha mostrado valores menores de reproducción y de tamaño individual cuando las condiciones no son óptimas y además hay una sustancia tóxica en el sistema.

En relación con las diferentes respuestas observadas en este ensayo crónico bajo condiciones subóptimas y óptimas, es interesante el estudio realizado por Rowe et al. (2009) que relaciona las tasas de reproducción y crecimiento con la energía disponible para los organismos.

Un organismo que se encuentra en condiciones óptimas o favorables para su desarrollo, es dotado de suficiente energía procedente del medio para poder realizar todas sus funciones vitales. Pero en el caso de que estas condiciones sean desfavorables, los organismos van a invertir la energía que les aporte el medio en sobrevivir, dando prioridad a funciones básicas para evitar el efecto perjudicial de los tóxicos. Esto significa emplear más energía en procesos de desintoxicación y reducir la energía disponible para las funciones de mantenimiento y reproducción, disminuyendo así el consumo de oxígeno y la tasa metabólica (Smolders et al. 2003, Rowe et al. 2009).

Con este razonamiento podemos explicar los resultados del ensayo crónico en condiciones subóptimas sobre $D$. magna desde un punto de vista bioenergético. Suponiendo que los individuos han estado empleando energía en procesos de desintoxicación, desviándolos de compartimentos destinados a crecimiento y reproducción.

Por lo tanto, el efecto negativo del fosfito de sodio sobre los individuos de D. magna podría estar condicionado por el estado del medio en el que se encuentre. Esto puede tratarse de un factor más a tener en cuenta a la hora de realizar las evaluaciones de riesgo ambiental de determinadas sustancias.

Los ecosistemas acuáticos naturales no siempre muestran las condiciones óptimas para todos los distintos organismos que habitan en ellos. Por lo que una cantidad de producto valorada en primera instancia y catalogada como no perjudicial, podría causar efectos negativos a determinadas especies y sus ciclos vitales, según las condiciones de las que se parta.

Este punto crítico ha sido resaltado por estudios previos (Elendt 1990, Elendt y Bias 1990) en condiciones de laboratorio y es lo que lleva a la necesidad de evaluaciones más complejas y ecológicamente más relevantes como son los estudios con microcosmos.

Los resultados obtenidos en los experimentos de microcosmos muestran que concentraciones de 5 $\mathrm{mg} / \mathrm{L}$ (B) y $10 \mathrm{mg} / \mathrm{L}$ (M) de fosfito de sodio dibásico no presentan un impacto negativo sobre la comunidad zooplanctónica, ni de forma directa debido a la baja toxicidad del compuesto, ni de forma indirecta mediante el posible efecto sobre el alimento disponible, ya que no generó incremento de la producción primaria del componente fitoplanctónico ni del componente asociado al perifiton.

En el caso de organismos zooplanctónicos, es a partir de concentraciones superiores a $61.26 \mathrm{mg} / \mathrm{L}$ de fosfito de sodio dibásico cuando se observan efectos letales en D. magna (EFSA 2013).

La ausencia de efectos tóxicos de los fosfitos sobre el fitoplancton ya había sido demostrada con anterioridad. Concentraciones entre 7.9 y $158.17 \mathrm{mg} / \mathrm{L}$ de fosfito potásico monobásico no presentaron efectos tóxicos que causaran mortalidad de las células para la microalga Chlamydomonas reinhardtii (LoeraQuezada et al. 2015). En el caso de la cianobacteria Microcystis aeruginosa, se ha observado que el fosfito parece ser inerte y no tóxico hasta concentraciones de $500 \mathrm{mg} / \mathrm{L}$ (Zhang et al. 2011). Por último, en concentraciones superiores a $61.26 \mathrm{mg} / \mathrm{L}$ de fosfito de sodio dibásico se observa la reducción de la tasa de crecimiento del alga Pseudokirchneriella subcapitata (EFSA 2013).

Las variaciones de fluorescencia (y por ende del fitoplancton) que se producen durante el periodo experimental se deben a cambios temporales de la comunidad planctónica dentro de los microcosmos, independientemente del tratamiento.

A partir de la semana tres del experimento se produce una disminución del fitoplancton, que podría estar ligada a la aparición de individuos macrozooplanctónicos de mayor tamaño y capacidad filtradora como Daphnia sp. y Alona sp., capaces de disminuir la abundancia del fitoplancton (Vignatti et al. 2007).

Al final del experimento se observa un incremento del fitoplancton, que podría deberse a la posibilidad de que los ostrácodos que aparecen en las últimas semanas del experimento, al ser organismos típicamente detritívoros, den lugar al reciclado de nutrientes de los microcosmos y hacen que estos estén disponibles de nuevo (Torres et al. 2013).

Esta respuesta estructural debería complementarse con aspectos funcionales que en la mayoría de los estudios quedan fuera de las evaluaciones ecotoxicológicas (Slijkerman et al. 2004, Pestana et al. 2009). 
Un cambio funcional nos habla de cambios en las tasas y patrones de procesos. Por ejemplo, obviar un indicador funcional tan relevante como la descomposición de hojarasca que regula el reciclado de nutrientes, comprometerá la evaluación y su uso con carácter predictivo (Gessner y Chauvet 2002).

Por tanto, un nuevo paso hacia evaluaciones más complejas para alcanzar un mayor realismo ecológico deberían considerar aspectos funcionales junto a estructurales.

\section{CONCLUSIÓN}

Al contrario de lo que en otros experimentos de microcosmos se ha encontrado, en los que la respuesta mostrada por la comunidad frente a la exposición a tóxicos ha sido mayor que la registrada en experiencias de ensayos uniespecíficos (AmiardTriquet 2015b), los resultados de nuestro estudio no han puesto de manifiesto dicha respuesta.

En este caso no ha tenido lugar ni la desaparición de especies sensibles ni el incremento de la dominancia de alguna de las más resistentes u oportunistas como los ostrácodos (Johnston y Roberts 2009).

Aunque no se hayan encontrado efectos negativos sobre la estructura de la comunidad zooplanctónica es necesario atender a la prudencia ya que el componente fitoplanctónico tan sólo ha sido evaluado mediante cambios en abundancia.

Los cambios que han podido tener lugar a nivel taxonómico no han sido evaluados, por lo que, aunque los resultados permitirían decir que el fosfito de sodio es una sustancia con bajo nivel de riesgo ambiental, estudios más profundos sobre cambios estructurales en la comunidad fitoplanctónica son necesarios.

\section{REFERENCIAS}

Amiard-Triquet C. (2015a). Introduction. En: Aquatic ecotoxicology. Advancing tools for dealing with emerging risks. Academic Press, Londres, Reino Unido, pp 1-24.

DOI: 10.1016/B978-0-12-800949-9.00001-2

Amiard-Triquet C. (2015b). How to improve toxicity assessment? From single-species tests to mesocosms and field studies. En: Aquatic ecotoxicology. Advancing tools for dealing with emerging risks. (C. Amiard-Triquet, J.C. Amiard y C. Mouneyrac Eds.) Academic Press, Londres, Reino Unido, pp127-152. DOI: 10.1016/B978-0-12-800949-9.00006-1
Barmentlo S.H., Schrama M., Hunting E.R., Heutink R., van Bodegom P.M., de Snoo G.R. y Vijver M.G. (2018). Assessing combined impacts of agrochemicals: Aquatic macroinvertebrate population responses in outdoor mesocosms. Sci. Total. Environ. 631-632, 341-47. DOI: 10.1016/j.scitotenv.2018.03.021

Becerra A.T. y Bravo X.L. (2010). La agricultura intensiva del poniente almeriense. Diagnóstico e instrumentos de gestión ambiental. M+A Revista Electrónica de Medioambiente 8, 18-40.

Bendis R.J. y Relyea R.A. (2016). Wetland defense: naturally occurring pesticide resistance in zooplankton populations protects the stability of aquatic communities. Oecol. 181 (2), 487-498.

DOI: $10.1007 / \mathrm{s} 00442-016-3574-9$

Campbell B.M., Beare D.J., Bennett E.M., Hall-Spencer J.M., Ingram J.S.I., Jaramillo F., Ortiz R., Ramankutty N., Sayer J.A. y Shindell D. (2017). Agriculture production as a major driver of the Earth system exceeding planetary boundaries. Ecol. Soc. 22 (4), 8.

DOI: $10.5751 /$ ES-09595-220408

Carpenter S.R., Caraco N.F., Correl D.L., Howarth R.W., Sharpley A.N. y Smith, V.H. (1998). Nonpoint pollution of surface waters with phosphorus and nitrogen. Ecol. Appl. 8 (3), 559-568. DOI:10.1890/10510761( 1998)008[0559:NPOSWW]2.9.CO;2

Ceacero J., Díaz Hernández J.L., Del Campo A.D. y Navarro Cerrillo, R.M. (2012). Evaluación temprana de técnicas de restauración forestal mediante fluorescencia de la clorofila y diagnóstico de vitalidad de brinzales de encina (Quercus ilex sub. ballota). Bosque 33 (2), 191-202. DOI: $10.4067 /$ S0717-92002012000200009

Chará-Serna A.M. y Richardson J.S. (2018). Chlorpyrifos interacts with other agricultural stressors to alter stream communities in laboratory microcosms. Ecol. Appl. 28 (1), 162-176. DOI: 10.1002/eap.1637

Del Arco A.I. (2015). Estudio ecotoxicológico de los efectos de agroquímicos en la estructura y funcionamiento de la comunidad planctónica. Tesis de Doctorado. Facultad de Ciencias Experimentales. Universidad de Jaén, Jaén, España, 278 pp. DOI: 10953/668

Díaz-Báez M.C., López M.C.B. y Ramírez A.J.E. (2004). Pruebas de toxicidad acuática: fundamentos y métodos. UNIBIBLOS. Universidad Nacional de Colombia. Bogotá Colombia, 116 pp.

Douville M., Jean K. y Houde M. (2016). Multitrophic aquatic toxicity of emerging brominated and phosphorus flame retardants. Fresen. Environ. Bull. 25, 3265-3271.

EFSA (European Food Safety Authority) (2013). Conclusion on the peer review of the pesticide risk assessment of the active substance disodium phosphonate. EFSA Journal 11(5), 1-50. DOI: 10.2903/j.efsa.2013.3213 
Elendt B.P. (1990). Influence of water composition on the chronic toxicity of 3,4-dichloroaniline to Daphnia magna. Water Res. 24, 1169-1172.

DOI: 10.1016/0043-1354(90)90181-5

Elendt B.P. y Bias W.R. (1990). Trace nutrient deficiency in Daphnia magna cultured in standard medium for toxicity testing. Effects of the optimization of culture conditions on life history parameters of Daphnia magna. Water Res. 24 (9), 1157-1167.

DOI: 10.1016/0043-1354(90)90180-E

Geitler L. (1922). Die mikrophyten-miocoenose der fontinalis-bestände des lunzer untersees und ihre abhängigkeit vom lichts. Int. Rev. Hydrobiol. 10, 683-691.

Gessner M.O. y Chauvet E. (2002). A case for using litter breakdown to assess functional stream integrity. Ecol. Appl. 12 (2), 498-510. DOI: 10.1890/1051-0761(2002) 012[0498:ACFULB]2.0.CO;2

Giraudo M., Dubé M., Lépine M., Gagnon P., Douville M. y Houde M. (2017). Multigenerational effects evaluation of the flame retardant tris (2-butoxyethyl) phosphate (TBOEP) using Daphnia magna. Aquat. Toxicol. 190, 142-149. DOI: 10.1016/j.aquatox.2017.07.003

González S., Perales H. y Salcedo M.O. (2008). La fluorescencia de la clorofila $a$ como herramienta en la investigación de efectos tóxicos en el aparato fotosintético de plantas y algas. REB 27 (4), 119-129.

Guerrero F., Parra G., Jiménez-Gómez F., Salazar C., Jiménez-Melero R., Galotti A., García-Muñoz E., Lendínez M.L. y Ortega F. (2006). Ecological studies in Alto Guadalquivir wetlands: a first step towards the application of conservation plans. Limnetica 25 (1-2), 95-106.

Jessen C., Bednarz V.N., Rix L., Teichberg M. y Wild C. (2015). Marine eutrophication En: Environmental indicators. (R.H. Armon, y O. Hänninen, Eds.). Springer, Dordrecht, Países Bajos, pp 177-206.

DOI: 10.1007/978-94-017-9499-2_11

Johnston E.L. y Roberts D.A. (2009). Contaminants reduce the richness and evenness of marine communities: a review and meta-analysis. Environ. Poll. 157, 17451752. DOI: 10.1016/j.envpol.2009.02.017

Junta de Andalucía (2016). Estación meteorológica de Jaén. Datos históricos de la estación meteorológica de Jaén de la red de estaciones agroclimáticas de la Comunidad Andaluza. Consejería de Agricultura, Pesca y Desarrollo Rural. Junta de Andalucía [en línea].

http://www.juntadeandalucia.es/agriculturaypesca/ifapa/ ria/servlet/FrontController?action=Static\&url=datos Historicos.jsp 27/04/2018

Karlberg L., Corell R.W., Fabry V.J., Hansen J., Walker B., Liverman D., Richardson K., Crutzen P. y Foley J. (2009b). Planetary boundaries: exploring the safe operating space for humanity. Ecol. Soc. 14 (2), 32. DOI: $10.5751 /$ ES-03180-140232
Kim E., Yoo S., Ro H., Han H., Baek Y., Eom I., Kim H., Kim P. y Choi K. (2013). Aquatic toxicity assessment of phosphate compounds. Environ. Health and Toxicol. 28, 1-7.

DOI: 10.5620/eht.2013.28.e2013002

Kützing F.T. (1833). Algarum aquae dulcis germanicarum. In commissis CA Schwertschkii. Gesellschaft Naturforschender, Halle, Alemania, 99 pp.

Li H., Su G., Zou M., Yu L., Letcher R.J., Yu H. y Liu C. (2015). Effects of tris (1, 3-dichloro-2-propyl) phosphate on growth, reproduction, and gene transcription of Daphnia magna at environmentally relevant concentrations. Environ. Sci. Technol. 49, 12975-12983. DOI: $10.1021 /$ acs.est.5b03294

LIFE03 (2006). Producción respetuosa con el medio ambiente en viticultura. Proyecto LIFE SINERGIA "Calidad y respeto al medio ambiente". Gobierno de La Rioja, La Rioja, España [en línea]. http://ec.europa.eu/environment/ life/project/Projects/index.cfm?fuseaction=home. showFile\&rep=file\&fil=SYNERGIA_viti.pdf $11 / 05 / 2018$

Loera-Quezada M.M., Leyva-González M.A., LópezArredondo D. y Herrera-Estrella L. (2015). Phosphite cannot be used as phosphorus source but is non-toxic for microalgae. Plant Sci. 231, 124-130.

DOI: $10.1016 /$ j.plantsci.2014.11.015

Lovatt C.J. y Mikkelsen R.L. (2006). Phosphite fertilizers: What are they? Can you use them? What can they do? Better Crops With Plant Food 90 (4), 11-13.

Mazzeo N., Clemente J., García-Rodríguez F., Gorga J., Kruk C., Larrea D., Meerhoff M., Quintans F., Rodríguez-Gallego L. y Scasso F. (2002). Eutrofización: causas, consecuencias y manejo. En: Perfil ambiental del Uruguay: 2002. (A. Domínguez y R.G. Prieto Eds.). Nordan Comunidad, Montevideo, Uruguay, 39-55 pp.

MA. (2005). Ecosystems and human well-being. Synthesis. Island Press. Washington, Estados Unidos de América. Millenium Assessment [en línea] https:// www.millenniumassessment.org/documents/document.356.aspx.pdf 11/05/18.

Moser T., Römbke J., Schallnass H. y Van Gestel C.A.M. (2007). The use of the multivariate principal response curve (PRC) for community level analysis: a case study on the effects of carbendazim on enchytraeids in terrestrial model ecosystems (TME). Ecotoxicology 16 (8), 573-583. DOI: 10.1007/s10646-007-0169-6

Nowell L.H., Normam J.E., Moran P.W., Martin J.D. y Stoned W.W. (2014). Pesticides toxicity index- a tool for assessing potential toxicity of pesticides mixtures to freshwater aquatic organisms. Sci. Total Environ. 476477, 144-157. DOI: 10.1016/j.scitotenv.2013.12.088 
OECD (1997). Environmental benefits from agriculture. Issues and policies. The Helsinki Seminar, Organization for Economic Co-operation and Development; Washington D. C. OECD Washington Center [distributor], París, Francia, 178 pp.

OECD 211 (2012). Daphnia magna Reproduction Test. Guideline for testing of chemicals. Organization for Economic Cooperation and Development, $21 \mathrm{pp}$. DOI: 10.1787/9789264185203-en

Parra G., Jiménez-Melero R. y Guerrero F. (2005). Agricultural impacts on Mediterranean wetlands: the effect of pesticides on survival and hatching rates in copepods. Annal. Limnol. 41 (3), 161-167.

DOI: 10.1051/limn:20054130161

Pestana J.L.T., Alexander A.C., Culp J.M., Baird D.J., Cessna, A.J. y Soares, A.M.V.M. (2009). Structural and functional responses of benthic invertebrates to imidacloprid in outdoor stream mesocosms. Environ. Pollut. 157, 2328- 2334. DOI: 10.1016/j.envpol.2009.03.027

Rowe D. C., Pierce C. L. y Wilton T. F. (2009). Fish assemblage relationships with physical habitat in wadeable Iowa streams. N. Am. J. Fish. Manage. 29 (5), 1314-1332. DOI: 10.1577/M08-192.1

Slijkerman D.M.E., Baird D.J., Conrad A., Jak R.G. y Van Straalen N.M. (2004). Assessing structural and functional plankton responses to carbendazim toxicity. Environ. Toxicol. Chem. 23, 455-462.

DOI: $10.1897 / 03-12$

Smith T.M. y Smith R.L. (2007). Ecología. 6 $6^{\text {a edición. }}$ Pearson Educación, Madrid, España, 776 pp.

Smolders R., De Boeck G. y Blust R. (2003). Changes in cellular energy budget as a measure of whole effluent toxicity in zebrafish (Danio rerio). Environ. Toxicol. Chem. 22 (4), 890-899 DOI: 10.1002/etc.5620220429

Swiecki T.J. y Bernhardt E.A. (2013). A reference manual for managing sudden oak death in California. Pacific Southwest Research Station. United States Department of Agriculture, Albany, California, EUA, 129 pp. DOI: $10.2737 /$ PSW-GTR-242

Torres R., Lugo C., Gordon E. y Suárez Villasmil L. (2013). Descomposición foliar in situ de Heliconia marginata en un humedal herbáceo (Barlovento, Venezuela). Polibotanica 35, 21-40.
Turner M.G., Gardner R.H. y O'Neil R.V. (2001). Landscape ecology in theory and practice: Pattern and process. Springer, Nueva York, EUA, 482 pp.

DOI: $10.1007 / 978-1-4939-2794-4$

Van den Brink P.J. y Ter Braak C.J.F. (1999). Principal responses curves: analysis of time-dependent multivariate responses of a biological community to stress. Environ. Toxicol. Chem. 18, 138-148.

DOI: $10.1002 /$ etc.5620180207

Velandia J., Viteri S.E., Rubio N.J. y Tovar F.O. (2012). Efecto del fosfito de potasio en combinación con el fungicida Metalaxyl + Mancozeb en el control de mildeo velloso (Peronospora destructor Berk) en cebolla de bulbo (Allium cepa L.). Revista Facultad Nacional de Agronomía Medellín 65 (1), 6317-6325.

Vignatti A., Echaniz S. y Martín M.C. (2007). El zooplancton de tres lagos someros de diferente salinidad y estado trófico en la región semiárida pampeana (Argentina). Gayana 71 (1), 34-48. DOI: $10.4067 / \mathrm{S} 0717-65382007000100005$

Vitousek P.M., Aber J.D., Howarth R.W., Likens G.E., Matson P.A., Schindler D.W., Schlesinger W.H. y Tilman D.G. (1997). Human alteration of the global nitrogen cycle: sources and consequences. Ecol. App. 7 (3), 737 750. DOI: 10.1890/1051-0761(1997)007[0737:HAO TGN]2.0.CO;2

Zafar M.I., Belgers J.D., Van Wijngaarden R.P.A., Matser A. y Van den Brink P.J. (2012). Ecological impacts of time-variable exposure regimes to the fungicide azoxystrobin on freshwater communities in outdoor microcosms. Ecotoxicology 21 (4), 1024-1038. DOI: $10.1007 / \mathrm{s} 10646-012-0856-9$

Zhang J., Geng J., Ren H., Luo J., Zhang A y Wang X. (2011). Physiological and biochemical responses of Microcystis aeruginosa to phosphite. Chemosphere 85 (8), 1325-1330.

DOI: $10.1016 /$ j.chemosphere.2011.07.049. 